\title{
Canadian Update on Surgical Procedures (CUSP) Urology Group consensus for intraoperative hemostasis during minimally invasive partial nephrectomy
}

Douglas C. Cheung ${ }^{1}$; Christopher J.D. Wallis ${ }^{1,2}$; Simon Possee $^{3}$; Camilla Tajzler ${ }^{4}$; Maurice Anidjar $^{5}$; Keith Barrett ${ }^{6}$; Tom Deklaj ${ }^{7}$; Darrel E. Drachenberg ${ }^{8}$; Howard Evans ${ }^{9}$; Christopher French $^{10}$; Geoffrey Gotto ${ }^{11}$; Jason Izard ${ }^{12}$; Umesh Jain ${ }^{1}$; Jun Kawakami ${ }^{11}$; Girish S. Kulkarni ${ }^{1}$; Jason Lee ${ }^{1}$; Jeffrey McCracken ${ }^{13}$; Thomas McGregor ${ }^{12}$; Patrick O. Richard ${ }^{14}$; Neal E. Rowe ${ }^{15}$; Robert Sabbagh $^{14}$; Blair St. Martin ${ }^{9}$; Stephanie Tatzel ${ }^{16}$; Naji Touma ${ }^{12}$; Hugues Widmer ${ }^{17}$; Joshua Wiesenthal ${ }^{18}$; Brian Yang ${ }^{19}$; Kevin C. Zorn ${ }^{17}$; Anil Kapoor ${ }^{4}$; Antonio Finelli ${ }^{1}$; Raj Satkunasivam $^{20}$; on behalf of the Canadian Update on Surgical Procedures (CUSP) Urology Group

${ }^{1}$ Division of Urology, Department of Surgery, University of Toronto, Toronto, ON, Canada; ${ }^{2}$ Department of Urology, Vanderbilt University Medical Center, Nashville, TN, United States; ${ }^{3}$ Division of Medicine, The Rotherham Foundation Trust, South Yorkshire, United Kingdom; ${ }^{4}$ Division of Urology, McMaster Institute of Urology, Hamilton, ON, Canada; ${ }^{5}$ Division of Urology, McGill University, Montreal, QC, Canada; ${ }^{6}$ Kitchener Urology Partners, Kitchener, ON, Canada; ${ }^{7}$ Department of Surgery (Urology), Western University (Windsor Regional Hospital), London, ON, Canada; ${ }^{8}$ Section of Urology, University of Manitoba, Winnipeg, MB Canada; ${ }^{9}$ Division of Urology, Department of Surgery, University of Alberta, AB, Canada; ${ }^{10}$ Discipline of Surgery (Urology), Memorial University, St. John's, NL, Canada; ${ }^{11}$ Division of Urology, Department of Surgery, University of Calgary, Calgary, AB, Canada; ${ }^{12}$ Department of Urology, Queen's University, Kingston, ON, Canada; ${ }^{13}$ Victoria Urology, Victoria, BC, Canada; ${ }^{14}$ Division of Urology, Department of Surgery, Centre Hospitalier Universitaire de Sherbrooke and Centre de Recherche du CHUS, Université de Sherbrooke, QC, Canada; ${ }^{15}$ Division of Urology, Department of Surgery, University of Ottawa, Ottawa, ON, Canada; ${ }^{16}$ Division of Urology, Department of Surgery, McMaster University (Niagara Health), Hamilton, ON, Canada; ${ }^{17}$ Section of Urology, Department of Surgery, University of Montreal Hospital Center (CHUM), Montreal, QC, Canada; ${ }^{18}$ Kelowna General Hospital, Kelowna, BC, Canada; ${ }^{19}$ Department of Urological Sciences, University of British Columbia, Vancouver, BC, Canada; ${ }^{20}$ Center for Outcomes Research, Houston Methodist Hospital, Medical Center, Houston, TX, United States

Support: Baxter International provided organizational support and sponsorship to attend the CUSP meeting, wherein meetings related to this specific study were held. Baxter had no role within the selection process of iterative surveys, or in the final consensus discussion, drafting, or critical review of the final manuscript.

Cite as: Can Urol Assoc J 2020 June 9; Epub ahead of print. http://dx.doi.org/10.5489/cuaj.6579

Published online June 9, 2020 


\begin{abstract}
Introduction: Partial nephrectomy remains the gold standard in the management of small renal masses. However, minimally invasive partial nephrectomy (MIPN) is associated with a steep learning curve, and optimal, standardized techniques for time-efficient hemostasis are poorly described. Given the relative lack of evidence, the goal was to describe a set of actionable guiding principles, through an expert working panel, for urologists to approach hemostasis without compromising warm ischemia or oncological outcomes.

Methods: A three-step modified Delphi method was used to achieve expert agreement on the best practices for hemostasis in MIPN. Panelists were recruited from the Canadian Update on Surgical Procedures (CUSP) Urology Group, which represent all provinces, academic and community practices, and fellowship- and non-fellowship-trained surgeons. Thirty-two (round 1) and 46 (round 2) panellists participated in survey questionnaires, and 22 attended the in-person consensus meeting.
\end{abstract}

Results: An initial literature search of 945 articles (230 abstracts) underwent screening and yielded 24 preliminary techniques. Through sequential survey assessment and in-person discussion, a total of 11 strategies were approved. These are temporally distributed prior to tumor resection (five principles), during tumor resection (two principles), and during renorrhaphy (four principles).

Conclusions: Given the variability in tumor size, depth, location, and vascularity, coupled with limitations of laparoscopic equipment, achieving consistent hemostasis in MIPN may be challenging. Despite over two decades of MIPN experience, limited evidence exists to guide clinicians. Through a three-step Delphi method and rigorous iterative review with a panel of experts, we ascertained a guiding checklist of principles for newly beginning and practicing urologists to reference. 


\section{Introduction}

Partial nephrectomy remains the gold standard in the management of small renal masses (cT1a) ${ }^{1-}$ ${ }^{4}$, and its utilization has been steadily increasing, particularly when performed by minimally invasive approaches. ${ }^{5,6}$ However, minimally invasive partial nephrectomy (MIPN) has a steep learning curve ${ }^{7}$, leading to considerable technical and procedural variation. ${ }^{8-10}$ Time-efficient yet effective hemostasis is critical during these procedures as peri-operative hemorrhage can lead to morbidity, increased costs, and must be carefully balanced with the risk of renal injury due to prolonged warm ischemia. ${ }^{11,12}$ The true role and benefit of hemostatic agents is unclear. Likely as a result, there is significant variation in the use of and type of hemostatic agents employed during MIPN. Acknowledging the relative scarcity of robust clinical evidence, we aimed to collate the opinions of expert academic and community urologists performing minimally invasive partial nephrectomy (MIPN) to achieve a consensus statement for best practices in achieving intra-operative hemostasis.

The Delphi method has been previously well described as a method of achieving expert consensus. ${ }^{13,14}$ It is a systematic approach to garner expert consensus opinion through sequential "rounds" involving the proposal of techniques and strategies, refinement of these guiding principles, and discussion to converge upon an accepted response. We have previously used this iterative process in determining quality indicators in renal cell carcinoma care. ${ }^{13} \mathrm{With}$ this approach and an expert working group of Canadian minimally invasive urologic surgeons, we sought to determine a set of evidence-based and actionable guiding principles for urologists to approach hemostasis during MIPN without compromising warm ischemia or oncological surgical margins.

\section{Methods}

\section{The Delphi method}

The 3-step Delphi method of consensus was used to achieve expert agreement on best practices for hemostasis in MIPN. A systematic literature search to identify potential techniques and strategies was completed, followed by a three round iterative approach (surveys and final group meeting) to achieve consensus. The MIPN procedure was divided into 3 distinct potential timeframes to examine procedural variability and developing consensus in the study: 1) prior to tumor resection, 2) during tumor resection, and 3) during renorrhaphy and following vascular unclamping.

\section{Panel selection}

Panelists were recruited from members of the Canadian Update on Surgical Procedures (CUSP) Urology Group which meets biannually for scientific and technical exchanges. CUSP members represent all provinces, academic and community practice settings. Overall, panelists had been in practice for a mean of 11 years (range: 2.5 to 21 years) and performed an average case volume of 
35 MIPN (range: 10-100 MIPN) per year. Most panelists were fellowship trained urologic surgeons. Panelists agreed to participate after project presentation at the 2017 CUSP meeting.

\section{Literature search}

We conducted a search of the literature using the search terms "partial nephrectomy" AND ("robotic" or "laparoscopic" or "minimally invasive") AND ("hemostasis" or "hemorrhage" or "bleeding" or "blood loss" or "hemostatic agents") using MEDLINE for relevant publications between January 2000 to August 2017. English language publications that were original research and review articles were screened for relevance to hemostasis in MIPN, including their manuscript references for completeness (DC, SP, and RS). Articles were excluded if they did not include a MIPN cohort, were editorials or abstracts only, did not include human subjects, did not discuss a hemostatic technique or agent, and/or did not include hemostasis as an outcome. A non-duplicated list of best principles for preliminary inclusion was tabulated for each timeframe listed. Review of the articles (DC, SP, and RS) and of the subsequent suggested techniques (all authors) was completed prior to round 1 .

\section{Rounds 1 and 2}

Following literature search, principles that were identified as relevant to each of "prior to tumor resection," "during tumor resection," and "during renorrhaphy" were reviewed by the authors, and a list of principles was then distributed via email mailing list to the CUSP membership (Round 1: 32 panellists; Round 2: 46 panellists). These were ranked according to Likert scale from 1 (not at all relevant) to 7 (extremely relevant) for achieving hemostasis in MIPN based on their clinical experience. All responses were recorded and graphed to demonstrate the frequency of distribution per item and ranking.

A score of 4 was defined as neither relevant nor irrelevant within the survey and did not count towards approval or rejection of a proposed technique. Approval and advancement required over $50 \%$ agreement of adopting the item (Likert score 5, 6, 7). Furthermore, a free text option was available at each stage for participants to suggest additional principles that had been omitted or overlooked, and to provide general comments. These were then carefully reviewed by the authors (DC and RS). Once duplicate suggestions and those not pertaining directly to hemostasis were excluded, free text suggestions were incorporated into the next survey iteration with the collated results from existing principles. In some cases, principles of hemostasis were combined for conciseness and clarity. A new questionnaire was then designed with the updated principles. This process was repeated following the second round.

\section{Round 3}

An in-person meeting was planned for November 2017 for third round review of the suggested principles. This was attended by 22 panelists as defined above. At this meeting, the results from rounds 1 and 2 were collated and presented. This included the principles that had advanced through the screening as well as justification for those which had not. Panelists were offered the 
opportunity to directly exclude any principles that they felt were not relevant via group discussion. At this meeting, it was felt that there should not be prioritization of principles as one may become more or less clinically relevant in any given case and subject to the clinical expertise of the operating surgeon.

In total, 32 panelists submitted responses to round 1 of the survey (28 fully completing the survey), 46 panelists submitted responses to round 2 of the survey (35 fully completing the survey), and 22 individuals attended the in-person meeting for round 3 . The full modified Delphi approach and the results from each round are summarized in Figure 1.

\section{Manuscript review}

The draft manuscript was then reviewed over email distribution as well as at the CUSP Urology Group biannual meeting November 2019 for final approval. This was attended by 18 CUSP panelists. Minor wording/clarity changes were made with no content changes to the final guiding principles.

\section{Results}

A total of 945 articles were identified as having potential relevance to hemostasis and MIPN. All articles were reviewed by the authors by title, and 230 were further screened for hemostatic principles by abstract. Of these, an initial 24 techniques of achieving hemostasis in MIPN were tabulated. These were distributed into 7 techniques prior to tumor resection, 8 techniques during tumor resection, and 9 techniques during renorrhaphy. These preliminary techniques and strategies were then distributed to expert urologists in the first round [Supplementary Appendix $1 \mathrm{~A} / 1 \mathrm{~B}]$.

Of the initial 24 principles, 11/24 were accepted and advanced to the second round and $13 / 24$ were rejected by over $50 \%$ agreement, while 7 new principles were proposed via free text by the group [Figure 1]. The authors then reviewed all the results prior to the creation of the second round survey.

For the second round, 17/18 principles were approved, 1/18 was rejected, and 3 principles were newly suggested [Supplementary Appendix 2A/2B]. These results were again reviewed and summarized in a similar fashion to the first round before advancing to the final in-person meeting.

All the final guiding principles were reviewed and discussed at the in-person meeting in round 3 [Table 1]. These are distributed across prior to tumor resection ( 5 principles), during tumor resection ( 2 principles), and during renorrhaphy ( 4 principles). The remainder of the principles were merged for clarity or excluded directly at the time of the meeting.

\section{Discussion}

At this time, despite nearly 2 decades of MIPN experience, very limited evidence exists to guide clinicians regarding techniques to optimize hemostasis during MIPN. We rigorously reviewed techniques for achieving hemostasis in MIPN using a 3-step Delphi method, including a 


\section{Intraoperative hemostasis during minimally invasive partial nephrectomy}

geographically diverse panel of Canadian experts in minimally invasive surgery from academic and community practice settings (CUSP). By systematically probing these techniques and strategies through an iterative approach, members of the survey and panel could provide detailed input at each stage regarding the inclusion of relevant techniques and the exclusion of those that were not. We outlined multiple timeframes within MIPN (prior to tumor resection, during tumor resection, and during renorrhaphy) to further improve the applicability and ease of utility of the final metrics. This will allow surgeons to quickly reference them as an evidence-based, concise, and actionable checklist for their approach.

In total, 24 principles were initially suggested from the literature search and an additional 10 were proposed during surveys, with 11 principles ultimately achieving overall group consensus through all three iterations. Of these, the majority of approved strategies (7/11: discontinuation of therapeutic anti-coagulation, selective clamping based on anatomy, targeting pneumoperitoneum, reducing the resection margin to tumor, use of a barbed stitch, use of gelatin-based sealant, and placing clips instead of knot tying sutures) were maintained across all rounds, highlighting the robust nature of the initial literature review and consistency throughout the selection process.

We recognize that different provinces within Canada may have conflicting availability of hemostatic agents. Of note, gelatin thrombin agents were specifically identified within the panel over fibrin sealants, oxidized cellulose polymer, and hemostatic patch, but may represent the panel's familiarity with these agents. In real world application, in the absence of randomized evidence stating superiority, this may depend on the operator's experience. Ultimately, within this area, there continues to be limited evidence towards their efficacy outside of animal models, single centre or single surgeon small retrospective case series, or only with selective agents/techniques ${ }^{15-23}$, and this study is the first to provide an approach on the utilization of these agents through the experience of expert urologic surgeons (CUSP).

In addition, individuals who are fellowship trained versus not, in academic versus community practice, and in teaching versus non-teaching centres may also having differing opinions towards the optimal techniques in hemostasis. Throughout our surveys and panel discussion, we were conscientious to include surgeons representative of a variety of practice settings where possible (although the majority were fellowship trained).

A few principles were directly excluded during the in-person meeting. In particular, the authors would like to highlight three of the principles that did not advance to the final consensus document: "early clipping of identifiable blood vessels," "unclamping the renal vein if bleeding was encountered," and "increasing the pneumoperitoneum with bleeding." In particular, the members of the panel stressed that the "early clipping of identifiable blood vessels" and "unclamping the renal vein in the occurrence of bleeding" were important, but represented basic surgical principles that did not require special mention so as to not dilute the quality of the remaining strategies. "Increasing the pneumoperitoneum" was felt to be captured within "achieving adequate pneumoperitoneum" prior to tumor resection. 
We believe that these methods represent an excellent foundational basis towards achieving hemostasis in MIPN. They are helpful principles designed to aid newly starting and currently practicing urologic surgeons, but are not an exhaustive or mandatory requirement. Additionally, these principles may also have extended applications in surgical education, standardization, quality-care benchmarking, and/or inclusion into surgical safety checklists in the future. Ultimately, while these guiding principles highlight important components of achieving hemostasis in MIPN, surgical planning must remain individualized to the tumour (i.e. nephrometry score), the patient, and the surgeon.

\section{Limitations}

There are several limitations in using the Delphi technique to achieve consensus. Although the iterative process is designed to generate and refine the proposed principles as much as possible, this remains limited by the composition of the panel. Our panel was chosen to represent diverse practice settings; however, some hemostatic agents were not available to all panel members and may not be accessible within your own locale. More vocal members of the panel also have the opportunity to influence the results of the discussion over other members, although this was not seen during our in-person meeting. Furthermore, given that our results and voting were anonymous in the initial surveys, this decreased the risk of bias from this metric.

\section{Conclusions}

Achieving hemostasis in MIPN can be a challenging task and limited evidence exists to date to guide clinicians. Through a 3-step Delphi method and rigorous iterative review with a panel of experts, we ascertained a guiding checklist of principles from which newly beginning and practicing urologists can refer to. Overall, the goal of this study is to identify commonly accepted principles of hemostasis to improve the quality of MIPN that is performed across Canada. 


\section{References}

1. Campbell, S., R. G. Uzzo, M. E. Allaf, et al. Renal Mass and Localized Renal Cancer: AUA Guideline. The Journal of urology 2017;198:520-9. http://www.ncbi.nlm.nih.gov/pubmed/28479239

2. Ljungberg, B., K. Bensalah, S. Canfield, et al. EAU guidelines on renal cell carcinoma: 2014 update. European urology 2015;67:913-24. http://www.ncbi.nlm.nih.gov/pubmed/25616710

3. Gill, I. S., M. Aron, D. A. Gervais, et al. Clinical practice. Small renal mass. The New England journal of medicine 2010;362:624-34. http://www.ncbi.nlm.nih.gov/pubmed/20164486

4. Finelli, A., N. Ismaila, B. Bro, et al. Management of Small Renal Masses: American Society of Clinical Oncology Clinical Practice Guideline. Journal of clinical oncology : official journal of the American Society of Clinical Oncology 2017;35:668-80. http://www.ncbi.nlm.nih.gov/pubmed/28095147

5. Alameddine, M., T. Koru-Sengul, K. J. Moore, et al. Trends in Utilization of Robotic and Open Partial Nephrectomy for Management of cT1 Renal Masses. European urology focus 2018; http://www.ncbi.nlm.nih.gov/pubmed/29325761

6. Pak, J. S., J. J. Lee, K. Bilal, et al. Utilization trends and outcomes up to 3 months of open, laparoscopic, and robotic partial nephrectomy. Journal of robotic surgery 2017;11:223-9. http://www.ncbi.nlm.nih.gov/pubmed/27804062

7. Aboumarzouk, O. M., R. J. Stein, R. Eyraud, et al. Robotic versus laparoscopic partial nephrectomy: a systematic review and meta-analysis. European urology 2012;62:102333. http://www.ncbi.nlm.nih.gov/pubmed/22771266

8. Dell'Atti, L., S. Scarcella, S. Manno, et al. Approach for Renal Tumors With Low Nephrometry Score Through Unclamped Sutureless Laparoscopic Enucleation Technique: Functional and Oncologic Outcomes. Clinical genitourinary cancer 2018;16:e1251-e6. http://www.ncbi.nlm.nih.gov/pubmed/30122517

9. Wille, A. H., M. Tullmann, J. Roigas, et al. Laparoscopic partial nephrectomy in renal cell cancer--results and reproducibility by different surgeons in a high volume laparoscopic center. European urology 2006;49:337-42; discussion 42-3. http://www.ncbi.nlm.nih.gov/pubmed/16413957

10. Carrion, D. M., Y. G. SA, J. G. Rivas, et al. The role of hemostatic agents in preventing complications in laparoscopic partial nephrectomy. Central European journal of urology 2017;70:362-7. http://www.ncbi.nlm.nih.gov/pubmed/29410886

11. Shin, J., K. Han, J. H. Kwon, et al. Clinical Results of Transarterial Embolization to Control Postoperative Vascular Complications after Partial Nephrectomy. The Journal of urology 2019; http://www.ncbi.nlm.nih.gov/pubmed/30395840 


\section{Intraoperative hemostasis during minimally invasive partial nephrectomy}

12. Alimi, Q., B. Peyronnet, P. Sebe, et al. Comparison of Short-Term Functional, Oncological, and Perioperative Outcomes Between Laparoscopic and Robotic Partial Nephrectomy Beyond the Learning Curve. Journal of laparoendoscopic \& advanced surgical techniques. Part A 2018;28:1047-52.

http://www.ncbi.nlm.nih.gov/pubmed/29664692

13. Wood, L., G. A. Bjarnason, P. C. Black, et al. Using the Delphi technique to improve clinical outcomes through the development of quality indicators in renal cell carcinoma. Journal of oncology practice 2013;9:e262-7. http://www.ncbi.nlm.nih.gov/pubmed/23943895

14. Pauyo, T., N. Verma, Y. Marwan, et al. Canadian Consensus for the Prevention of Blood Loss in Spine Surgery. Spine 2017;42:E50-E5. http://www.ncbi.nlm.nih.gov/pubmed/27172287

15. Imkamp, F., Y. Tolkach, M. Wolters, et al. Initial experiences with the Hemopatch(R) as a hemostatic agent in zero-ischemia partial nephrectomy. World journal of urology 2015;33:1527-34. http://www.ncbi.nlm.nih.gov/pubmed/25239500

16. Hassouna, H. A. and R. Manikandan. Hemostasis in laparoscopic renal surgery. Indian journal of urology : IJU : journal of the Urological Society of India 2012;28:3-8. http://www.ncbi.nlm.nih.gov/pubmed/22557709

17. Harty, N. J., A. Moinzadeh, S. Flacke, et al. Temporary targeted hemostasis to facilitate bloodless partial nephrectomy. Urology 2011;78:1435-41. http://www.ncbi.nlm.nih.gov/pubmed/22137714

18. Galanakis, I., N. Vasdev and N. Soomro. A review of current hemostatic agents and tissue sealants used in laparoscopic partial nephrectomy. Reviews in urology 2011;13:131-8. http://www.ncbi.nlm.nih.gov/pubmed/22110396

19. Antonelli, A., A. Minervini, A. Mari, et al. TriMatch comparison of the efficacy of FloSeal versus TachoSil versus no hemostatic agents for partial nephrectomy: results from a large multicenter dataset. International journal of urology : official journal of the Japanese Urological Association 2015;22:47-52. http://www.ncbi.nlm.nih.gov/pubmed/25139104

20. Binsaleh, S. Evaluation of hemostatic AIDS in laparoscopic nephron-sparing surgery. TheScientificWorldJournal 2011;11:133-41. http://www.ncbi.nlm.nih.gov/pubmed/21258756

21. Cohen, J., G. Jayram, J. K. Mullins, et al. Do fibrin sealants impact negative outcomes after robot-assisted partial nephrectomy? Journal of endourology 2013;27:1236-9. http://www.ncbi.nlm.nih.gov/pubmed/23883149

22. Bahler, C. D., H. T. Dube, K. J. Flynn, et al. Feasibility of omitting cortical renorrhaphy during robot-assisted partial nephrectomy: a matched analysis. Journal of endourology 2015;29:548-55. http://www.ncbi.nlm.nih.gov/pubmed/25616087 
Intraoperative hemostasis during minimally invasive partial nephrectomy

23. Jeon, S. H., S. Jung, H. S. Son, et al. The unidirectional barbed suture for renorrhaphy during laparoscopic partial nephrectomy: Stanford experience. Journal of laparoendoscopic \& advanced surgical techniques. Part A 2013;23:521-5. http://www.ncbi.nlm.nih.gov/pubmed/23414123 
Figures and Tables

Fig. 1. Modified Delphi technique process

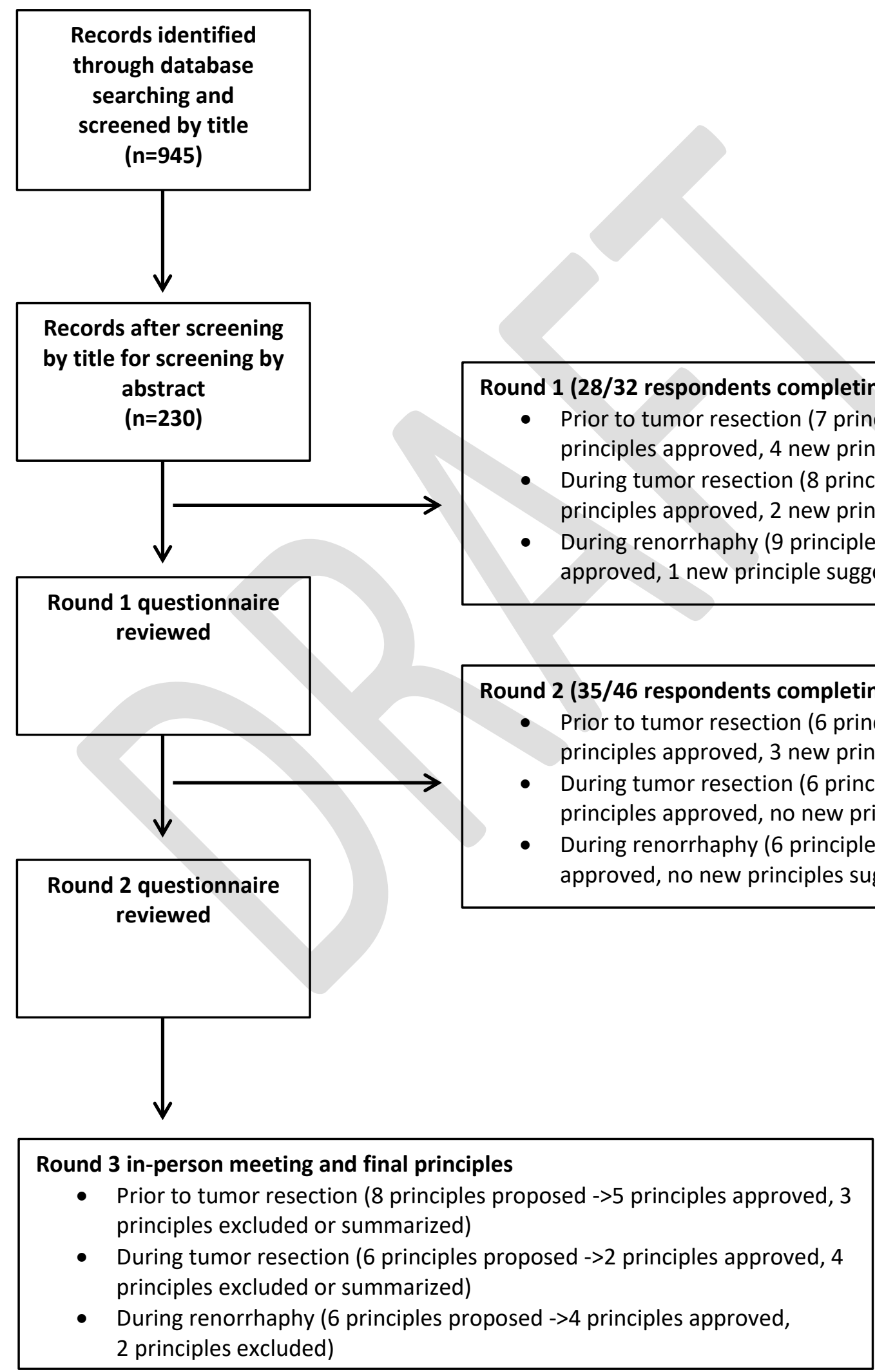




\section{Table 1. Final approved considerations for achieving hemostasis in minimally invasive partial nephrectomy}

\section{Final approved techniques and strategies}

Prior to tumor resection

1. Discontinuation of therapeutic anticoagulation, excluding ASA for high-risk individuals

2. Ensuring adequate renal mobilization and hilar dissection

3. Consider selective arterial clamping based on anatomy

4. Targeting adequate pneumoperitoneum pressure

5. Pre-clamp time out:

a. Prior preparation of all sutures including safety sutures ahead of time (i.e., number, bolster, type, length)

b. Availability of hemostatic agent ready for use

c. During robotic partial nephrectomy, ensure trocars are visible and tract is clear to facilitate instrument change if needed

\section{During tumor resection}

1. Reducing distance of resection margin from tumor (e.g., staying closer to the tumour)

2. Assistant or surgeon to apply pressure on bleeding areas

\section{During renorrhaphy}

1. Consider using a barbed stitch instead of traditional absorbable suture for hemostatic running stitch

2. Consider the use of gelatin thrombin agents if available

3. Placing clips (e.g., sliding clip technique) over knot tying of sutures

4. Early unclamping to identify and suture ligate significant arterial bleeding prior to apposition of the parenchyma 
Intraoperative hemostasis during minimally invasive partial nephrectomy

\begin{tabular}{|c|c|c|}
\hline \multicolumn{3}{|c|}{ Supplementary Appendix 1A. Suggested techniques and strategies from round 1} \\
\hline Prior to tumor resection & During tumor resection & During renorrhaphy \\
\hline $\begin{array}{l}\text { 1. Clamping artery + } \\
\text { vein vs. artery alone } \\
\text { 2. Controlled } \\
\text { hypotension } \\
\text { 3. Robotic vs. Lap } \\
\text { 4. Discontinuation of } \\
\text { anti-platelet agents } \\
\text { 5. Antifibrinolytics } \\
\text { 6. En-bloc clamping vs. } \\
\text { bulldogs } \\
\text { 7. Intra-operative } \\
\text { doppler for flow } \\
\text { Newly suggested: } \\
\text { - Renal mobilization and } \\
\text { hilar dissection } \\
\text { - Selective arterial } \\
\text { clamping } \\
\text { - Adequate } \\
\text { pneumoperitoneum } \\
\text { - Prior suture preparation }\end{array}$ & $\begin{array}{l}\text { 1. Enucleation } \\
\text { 2. Early clipping of } \\
\text { vessels } \\
\text { 3. Reducing resection } \\
\text { margin to tumor } \\
\text { (staying close to } \\
\text { tumor) } \\
\text { 4. Increasing } \\
\text { pneumoperitoneum } \\
\text { 5. Electrocautery of } \\
\text { resection bed } \\
\text { 6. Electrocautery } \\
\text { resection } \\
\text { 7. Argon beam } \\
\text { coagulator } \\
\text { 8. Ultrasonic/Harmonic } \\
\text { Newly suggested: } \\
\text { Assistant to apply } \\
\text { pressure } \\
\text { - Unclamping vein if } \\
\text { bleeding }\end{array}$ & $\begin{array}{l}\text { 1. Barbed stitch/Vloc } \\
\text { 2. Fibrin glue/sealants } \\
\text { (Tisseel, Evicel) } \\
\text { 3. Gelatin thrombin } \\
\text { agents (Floseal, } \\
\text { Surgiflo) } \\
\text { 4. Oxidized cellulose } \\
\text { polymer (Surgicel, } \\
\text { Surgifoam) } \\
\text { 5. Hemostatic patch } \\
\text { (Hemopatch) } \\
\text { 6. Bolster } \\
\text { 7. Placing clips over } \\
\text { knot tying } \\
\text { 8. Re-approximation of } \\
\text { Gerota's Fascia } \\
\text { 9. Placement of a drain } \\
\text { Newly suggested: } \\
\text { - Early unclamping and } \\
\text { suture ligate arterial } \\
\text { bleeders }\end{array}$ \\
\hline
\end{tabular}

Bold indicates agreement/approval of the principle for next round. 
Intraoperative hemostasis during minimally invasive partial nephrectomy

\begin{tabular}{|c|c|}
\hline Supplementary Appendix 1B. Truncated r & ponses round 1 \\
\hline Truncated response & Reasoning \\
\hline $\begin{array}{l}\text { Prior to tumor resection: } \\
\text { "Consideration of use of bulldog clamps if } \\
\text { hilar anatomy demonstrates multiple vessels. } \\
\text { Use of en-bloc clamp may not occlude } \\
\text { multiple arteries, while allowing the vein to } \\
\text { be clamped" } \\
\text { "Ensuring good correlation between CT } \\
\text { findings of number of vessels and } \\
\text { intraoperative findings" }\end{array}$ & Summarized within existing principles \\
\hline $\begin{array}{l}\text { During tumor resection: } \\
\text { "Running suture layer of the base layer" }\end{array}$ & Captured within "during renorrhaphy" \\
\hline $\begin{array}{l}\text { During renorrhaphy: } \\
\text { "Technique of suturing should be mastered" } \\
\text { "2 layer closure with barbed suture, when } \\
\text { possible" } \\
\text { "Don't place parenchymal (1st layer) sutures } \\
\text { too deep (can hit larger vessels)" } \\
\text { "Use of hemostatic agents should be PRN } \\
\text { only" } \\
\text { "Lysine analogs" } \\
\text { "Bringing cortex together. Not putting } \\
\text { surgicel in the defect." } \\
\text { "Horizontal mattress closure" }\end{array}$ & $\begin{array}{l}\text { Summarized within existing principles or } \\
\text { excluded as within basic surgical skills }\end{array}$ \\
\hline
\end{tabular}




\begin{tabular}{|c|c|c|}
\hline \multicolumn{3}{|c|}{ Supplementary Appendix 2A. Suggested techniques and strategies from round 2} \\
\hline Prior to tumor resection & During tumor resection & During renorrhaphy \\
\hline $\begin{array}{l}\text { 1. Clamping artery + } \\
\text { vein vs. artery alone } \\
\text { 2. Discontinuation of } \\
\text { anti-platelet agents } \\
\text { 3. Renal mobilization } \\
\text { and hilar dissection } \\
\text { 4. Selective arterial } \\
\text { clamping } \\
\text { 5. Adequate } \\
\text { pneumoperitoneum } \\
\text { 6. Prior suture } \\
\text { preparation } \\
\text { Newly suggested: } \\
\text { - Robotic - ensure } \\
\text { trocars/tract visible if } \\
\text { instrument change } \\
\text { needed } \\
\text { - Having Gelatin based } \\
\text { sealant ready } \\
\text { - Having Vloc ready }\end{array}$ & $\begin{array}{l}\text { 1. Enucleation } \\
\text { 2. Early clipping of } \\
\text { vessels } \\
\text { 3. Reducing resection } \\
\text { margin to tumor } \\
\text { (staying close to } \\
\text { tumor) } \\
\text { 4. Increasing } \\
\text { pneumoperitoneum } \\
\text { 5. Assistant to apply } \\
\text { pressure } \\
\text { 6. Unclamping vein if } \\
\text { bleeding } \\
\text { Modification to existing: } \\
\text { - Apply pressure to } \\
\text { bleeding sites }\end{array}$ & 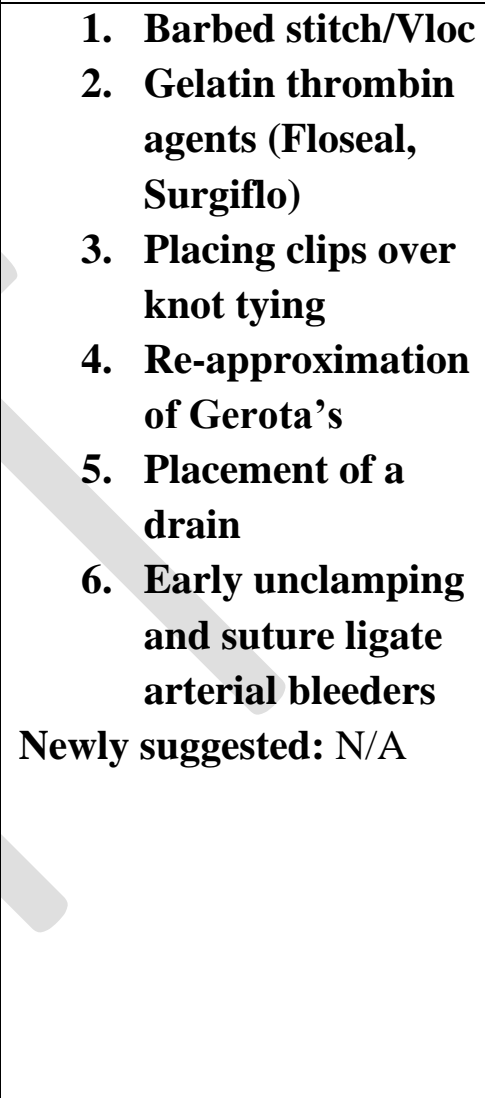 \\
\hline
\end{tabular}

Bold indicates agreement/approval of the principle for next round. 
Intraoperative hemostasis during minimally invasive partial nephrectomy

\begin{tabular}{|l|l|}
\hline Supplementary Appendix 2B. Truncated responses round 2 \\
\hline Truncated response & Reasoning \\
\hline $\begin{array}{l}\text { Prior to tumor resection: } \\
\text { "Satinsky if heminephrectomy, selective } \\
\text { artery alone for small amenable lesions, artery } \\
\text { alone for non-complex cases" }\end{array}$ & Summarized within existing principles \\
\hline $\begin{array}{l}\text { During tumor resection: } \\
\text { "Pressure on the parenchyma with an } \\
\text { instrument the surgeon controls" } \\
\text { "Placing 2 bulldogs on artery" }\end{array}$ & Summarized within existing principles \\
\hline $\begin{array}{l}\text { During renorrhaphy: } \\
\text { "Reduce pneumoperitoneum and bring BP to } \\
\text { normal to 'test' closure before finishing" }\end{array}$ & $\begin{array}{l}\text { Summarized within existing principles or } \\
\text { "Oved as within basic surgical skills }\end{array}$ \\
$\begin{array}{l}\text { "Oversew collecting system separately from } \\
\text { other stitches" }\end{array}$ & \\
\hline
\end{tabular}

